\title{
CONSUMO HÍDRICO E COEFICIENTE DE CULTURA DA VIDEIRA NA REGIÃO DE PETROLINA, PE
}

\author{
Antônio Heriberto de Castro Teixeira ${ }^{1}$, Pedro Vieira de Azevedo ${ }^{2}$, Bernardo Barbosa da Silva ${ }^{2}$ \\ \& José Monteiro Soares ${ }^{1}$
}

\begin{abstract}
RESUMO
Medidas do saldo de radiação, fluxo de calor no solo, gradientes de temperatura e de pressão do vapor, obtidas em experimento de campo conduzido no Campo Experimental de Bebedouro, pertencente à Embrapa Semi-árido, em Petrolina, PE, no período de 03/06 a 11/09/94, foram utilizadas no cálculo da evapotranspiração da cultura da videira $\left(E_{c}\right)$ pelo método do balanço de energia baseado na Razão de Bowen. Para o cálculo da evapotranspiração de referência $\left(\mathrm{ET}_{\mathrm{o}}\right)$ utilizaram-se os métodos de Penman-Monteith e do Tanque Classe A. Com base nos valores de $\mathrm{ET}_{\mathrm{c}}$ e de $\mathrm{ET}_{\mathrm{o}}$, determinou-se o coeficiente de cultura $(\mathrm{kc})$ ao longo dos subperíodos de brotação das gemas, floração, chumbinho e maturação dos frutos. Os resultados indicam que, nas condições de clima e solo do experimento, a evapotranspiração da cultura $\mathrm{ET}_{\mathrm{c}}$ variou de $2,8 \mathrm{~mm} \mathrm{dia}^{-1}$ aos 18 dias após a poda a 7,0 $\mathrm{mm} \mathrm{dia}^{-1}$, aos 94 dias após a poda (subperíodo de chumbinho), decrescendo em seguida para atingir 4,4 mm dia ${ }^{-1}$ aos 117 dias após a poda (final do subperíodo de maturação dos frutos). O consumo hídrico da cultura ao longo de todo o ciclo produtivo foi da ordem de 503,0 $\mathrm{mm}$. Os valores do coeficiente de cultura variaram de 0,65 a 1,15 , de acordo com o método de cálculo da $\mathrm{ET}_{0}$, porém se mostraram superiores aos recomendados pela FAO.
\end{abstract}

Palavras-chave: evapotranspiração, balanço de energia, coeficiente de cultura, Vitis vinifera L.

\section{WATER CONSUMPTION AND CROP COEFFICIENT OF GRAPE VINE IN THE REGION OF PETROLINA, PERNAMBUCO STATE, BRAZIL}

\begin{abstract}
Measurements of net radiation, soil heat flux, air temperature and water vapor pressure gradients were used to evaluate crop evapotranspiration $\left(\mathrm{ET}_{\mathrm{c}}\right)$ by the energy balance method in grape vine crop. The reference evapotranspiration $\left(\mathrm{ET}_{\mathrm{o}}\right.$ ) was obtained by the methods of Penman-Monteith and Class A pan evaporation. Daily values of the crop coefficient $\left(\mathrm{kc}=\mathrm{ET}_{\mathrm{c}} / \mathrm{ET}_{\mathrm{o}}\right)$ were obtained throughout the phenological phases. The results showed that the mean daily values of $\mathrm{ET}_{\mathrm{c}}$ increased from $2.8 \mathrm{~mm}$ day $^{-1}$ at the $18^{\text {th }}$ day after pruning to $7.0 \mathrm{~mm}$ day $^{-1}$, at the $94^{\text {th }}$ day after pruning. After this, values decreased to $4.4 \mathrm{~mm} \mathrm{day}^{-1}$, at the $117^{\text {th }}$ day after pruning. In the whole growing period $\mathrm{ET}_{\mathrm{c}}$ accumulated was found to be $503.0 \mathrm{~mm}$. Crop coefficient values were different, according to the method used to calculate $\mathrm{ET}_{\mathrm{o}}$ but, in general, they were higher than those recommended by FAO.
\end{abstract}

Key words: evapotranspiration, energy balance, crop coefficient, Vitis vinifera L.

\footnotetext{
${ }^{1}$ Pesquisador, M.Sc., Embrapa Semi-Árido, CP 23, CEP 56300 - 000, Petrolina, PE. Fone: (081) 862 1711, Fax: (081) 8621744. E-mail: heribert@cpatsa.embrapa.br, monteiro@cpatsa.embrapa.br

${ }^{2}$ Professor, Ph.D, Universidade Federal da Paraíba, Av. Aprígio Veloso 882, Bodocongó, CEP 58109 - 970, Campina Grande, PB. Fone: (083) 333 1000, Fax: (083) 310 1202, E-mail: pvieira@dca.ufpb.br, bernardo@ dca.ufpb.br
} 


\section{INTRODUÇÃO}

O potencial de cultivo da cultura da videira na região do Submédio São Francisco tem sido incrementado a cada ano, por iniciativas públicas e privadas, visando às demandas dos mercados interno e externo. Este crescimento das áreas cultivadas com videira, na região do Submédio São Francisco, também é decorrente das condições climáticas altamente favoráveis à produção de uva de mesa e à irrigação, que compensa a heterogeneidade do regime pluviométrico desta região (Teixeira \& Azevedo, 1996).

Tanto a deficiência como o excesso hídrico afetam de maneira marcante o comportamento dos estádios fenológicos da cultura da videira. A deficiência, quando ocorre durante o período inicial de crescimento das bagas, proporciona redução na multiplicação celular e, quando acontece durante a maturação, condiciona redução no tamanho das células e, conseqüentemente, a redução do tamanho das bagas, além de favorecer a queima dos frutos, pelo sol. Na fase final de maturação das bagas o consumo hídrico da videira diminui (Vaadia \& Kasimatis, 1961, apud Winkler et al., 1974). O excesso hídrico, combinado com temperaturas elevadas, torna a cultura da videira muito susceptível a doenças fúngicas e pragas (Winkler et al., 1974). Teixeira \& Azevedo (1996) constataram que, para uma boa produtividade da cultura da videira, é recomendável que o desenvolvimento vegetativo da planta ocorra sob condições de déficit hídrico e que as necessidades hídricas de cada fase fenológica sejam satisfeitas através da irrigação; no entanto, a escolha do sistema de irrigação depende de uma série de fatores técnicos, econômicos e culturais, mas o gotejamento e a microaspersão se destacam por suas vantagens.

O consumo hídrico de um parreiral é uma função complexa dos balanços hídrico do solo e de energia da superfície cultivada (Heilman et al., 1994). Dentre os métodos de determinação da evapotranspiração destaca-se o do balanço de energia, que se baseia no princípio de conservação de energia, em que a energia disponível, geralmente associada à diferença entre o saldo de radiação (Rn) e o fluxo de calor no solo (G), é repartida entre os processos de evapotranspiração ou calor latente (LE) e as trocas devidas ao calor sensível do ar $(\mathrm{H})$. Baseado nesse princípio e na Razão de Bowen (b), que relaciona os fluxos H e LE, Tanner (1960) apresentou um modelo de estimativa da evapotranspiração de culturas, utilizando a forma simplificada da equação do balanço de energia. Na cultura da videira este método foi empregado por Mathias \& Coates (1986), Oliver \& Sene (1992), Heilman et al. (1994) e Teixeira et al. (1997).

A razão entre a evapotranspiração máxima da cultura e a evapotranspiração de referência origina os coeficientes de cultura, que dependem do estádio de desenvolvimento da cultura, do sistema de irrigação, da configuração de plantio e das condições meteorológicas reinantes. Esses coeficientes podem ser utilizados quando já estiverem sido determinados para a cultura de interesse, na estimativa da evapotranspiração da cultura, necessitando-se apenas de dados meteorológicos referentes à demanda atmosférica. A obtenção dos coeficientes de cultura é feita sob condições de não limitação hídrica, ou de qualquer outro fator climático ou fisiológico (Wright, 1988).
De acordo com Winkler et al. (1974) para parreirais californianos, o consumo hídrico da videira durante todo o seu ciclo varia de 405 a 1370 mm, enquanto Doorenbos \& Kassam (1979) afirmaram que, de maneira geral, os requerimentos hídricos variam de 500 a $1200 \mathrm{~mm}$, dependendo do clima, do solo, da variedade e do manejo cultural.

Para a cultura da videira variedade Concord, Doorenbos \& Kassam (1979) determinaram, no Hemisfério Norte, para condições de irrigações não freqüentes, solo seco na maior parte do tempo de avaliação e cobertura do solo de 30 - 35\% (sistema de condução em espaldeira) um conjunto de coeficientes de cultura que variaram de 0,25 a 0,75 , dependendo do estádio fenológico da videira. De acordo com Ávila Netto (1997) o consumo de água da cultura da videira variedade Itália, sob irrigação por gotejamento, na região do Submédio São Francisco, determinado pelo método do balanço de água no solo, para o período compreendido entre 13.05 a 11.08.96, foi da ordem de 332,8 mm, cujos valores de Kc aos 18 dias após a poda era de 0,62 , evoluiu acentuadamente atingindo o valor máximo de 0,74 no subperíodo de desenvolvimento das bagas, quando então passou a decrescer gradativamente, tendo alcançado o valor mínimo de 0,50 , por ocasião do final do subperíodo de maturação dos frutos, os quais se assemelham aos valores obtidos por Doorenbos \& Pruitt (1977) apud Ávila Netto (1997).

O presente trabalho objetivou determinar o consumo hídrico e o coeficiente de cultura para diferentes fases fenológicas da cultura da videira, nas condições edafoclimáticas da região do Submédio São Francisco.

\section{MATERIAL E MÉTODOS}

O experimento de campo foi conduzido no Campo Experimental de Bebedouro, pertencente à Embrapa Semi-Árido, localizada no município de Petrolina, PE (Latitude 0909'S, Longitude $40^{\circ} 24^{\prime} \mathrm{W}$ e altitude $365,5 \mathrm{~m}$ ). O clima da região é do tipo BSwh', segundo a classificação de Köeppen, correspondendo a uma região climaticamente árida, sendo a quadra chuvosa de janeiro a abril.

A cultura estudada foi a videira (Vitis vinifera $L$.) $\mathrm{cv}$. Itália, com três anos de idade, conduzida no sistema de latada, em Latossolo Vermelho-Amarelo, num espaçamento de 4 × $2 \mathrm{~m}$, sob irrigação por microaspersão, compreendendo emissores Dansprinkler mod. 2001 invertidos e suspensos na latada, vazão média de $38,65 \mathrm{~L} \mathrm{~h}^{-1}$, sob pressão de serviço de 1,5 atm, com um microaspersor para cada duas plantas. As lâminas líquida e bruta de irrigação aplicadas durante o ciclo de produção foram da ordem de 518,1 e de $843,2 \mathrm{~mm}$, respectivamente. A umidade do solo (\% umidade em volume, obtida pelo método gravimétrico) ficou em torno de 10, 12 e 14\% nas profundidades de 30, 60 e 90 $\mathrm{cm}$, respectivamente, valores esses bastante próximos aos da capacidade de campo das respectivas profundidades. O estudo foi realizado no período compreendido entre a poda de produção e a colheita dos frutos (03/06 a 11/09/1994).

Para determinação da evapotranspiração da cultura $\left(\mathrm{ET}_{\mathrm{c}}\right)$ foram realizadas medições do saldo de radiação $(\mathrm{Rn})$, do fluxo de calor no solo $(\mathrm{G})$ e dos gradientes de temperatura e pressão do vapor, em dois níveis acima da latada, sendo os dados armazenados num sistema de aquisição automática de dados (Datalogger Licor). 
$\mathrm{O}$ balanço de energia realizado teve por base medições do saldo de radiação $\mathrm{Rn},\left(\mathrm{W} \mathrm{m}^{-2}\right)$, do fluxo de calor no solo $\mathrm{G}$, $\left(\mathrm{W} \mathrm{m}{ }^{-2}\right)$ e dos gradientes de temperatura do ar $\left({ }^{\circ} \mathrm{C}\right)$ e pressão de vapor d'água $(\mathrm{kPa})$ que, combinados adequadamente, possibilitam estimar-se o fluxo de calor latente LE ( $\left.\mathrm{W} \mathrm{m}^{-2}\right)$; para tanto, utilizou-se o método da Razão de Bowen (Bowen, 1926; Tanner, 1960), conforme as seguintes expressões:

$$
L E=\frac{-(R n+G)}{1+\beta}
$$

sendo:

$$
\beta=\frac{H}{L E}=\frac{p_{O} \cdot c_{P}}{L . \varepsilon}\left(\frac{K h}{K v}\right) \frac{\partial T / \partial Z}{\partial e / \partial Z}=\gamma\left(\frac{K h}{K v}\right) \frac{\Delta T}{\Delta e}
$$

em que b é a razão de Bowen, $\mathrm{H}\left(\mathrm{W} \mathrm{m}^{-2}\right)$ é fluxo de calor sensível, $\mathrm{p}_{\mathrm{o}}$ é a pressão atmosférica local $(\mathrm{kPa}), \mathrm{c}_{\mathrm{p}}$ é o calor específico do ar a pressão constante $\left(\mathrm{J} \mathrm{kg}^{-1}{ }^{\circ} \mathrm{C}^{-1}\right)$, L é o calor latente de vaporização da água $\left(\mathrm{J} \mathrm{kg}^{-1}\right)$, eé razão entre as massas moleculares da água e do ar seco, Kh e Kv são os coeficientes de difusão turbulenta do calor sensível e do vapor d'água, respectivamente, DT e De são as diferenças de temperatura do $\operatorname{ar}\left({ }^{\circ} \mathrm{C}\right)$ e pressão de vapor d'água $(\mathrm{kPa})$, medidas a 0,5 e $1,5 \mathrm{~m}$ acima da copa, e gé a constante psicrométrica. Deve-se observar que, ao se realizar o balanço de energia, $\mathrm{Rn}+\mathrm{LE}+\mathrm{G}+\mathrm{H}=0$, e que os fluxos são positivos quando dirigidos ao dossel da cultura. Neste sentido, durante o período diurno são negativos os fluxos $\mathrm{G}$ e LE; no caso de $\mathrm{H}$, o mesmo será positivo sempre que a cultura estiver mais fria que o ar. Para se obter a água evapotranspirada pela cultura $(\mathrm{em} \mathrm{mm})$ dividiu-se o fluxo de calor latente (LE) pelo calor latente de vaporização da água, que é função da temperatura do ar e vale cerca de $2,5 \mathrm{MJ} \mathrm{kg}^{-1}$. Foram considerados os valores do balanço de energia correspondentes ao período diurno e sempre que $\mathrm{Rn}$ era maior que zero.

Para a determinação da evapotranspiração de referência $\left(\mathrm{ET}_{\mathrm{o}}\right.$ ) foram utilizados os métodos de Penman-Monteith (Allen et al., 1994) e do Tanque Classe A, enquanto para o cálculo de $\mathrm{ET}_{\mathrm{o}}$ pelo método de Penman-Monteith, utilizaram-se dados meteorológicos obtidos na estação agrometeorológica junto à área experimental, considerando-se uma cultura hipotética, com altura de $0,12 \mathrm{~m}$, resistência aerodinâmica da superfície de $70 \mathrm{~s} \mathrm{~m}^{-1}$ e coeficiente de reflexão de 0,23 (Allen et al., 1994; Sediyama, 1996) utilizando-se a seguinte equação:

$$
\mathrm{ET}_{\mathrm{o}}=\frac{0,408 \Delta(R n-G)+\gamma \frac{900}{T+273} U 2(e a-e d)}{\Delta+\gamma(1+0,34 U 2)}
$$

em que $\mathrm{ET}_{0}$ é a evapotranspiração de referência em mm, Rn é o saldo de radiação à superfície, em $\mathrm{MJ} \mathrm{m}^{-2}$, G é o fluxo de calor sensível no solo, em $\mathrm{MJ} \mathrm{m}^{-2}$, Té a temperatura média do ar $\left({ }^{\circ} \mathrm{C}\right)$, U2 é a velocidade do vento a $2 \mathrm{~m}$ de altura $\left(\mathrm{m} \mathrm{s}^{-1}\right)$, (ea - ed) refere-se ao déficit de pressão do vapor $(\mathrm{kPa}), \mathrm{D}$ corresponde à declividade da curva de pressão de vapor de saturação $\left(\mathrm{kPa}^{\circ} \mathrm{C}^{-1}\right)$ e 900 é um fator de conversão. Com relação ao método do Tanque Classe A, utilizou-se a expressão seguinte:

$$
\mathrm{ET}_{\mathrm{o}}=\mathrm{Eo} \cdot \mathrm{Kp}
$$

sendo Eo a evaporação do Tanque Classe A $\left(\mathrm{mm} \mathrm{dia}^{-1}\right)$ e Kp o coeficiente de tanque obtido conforme Doorenbos \& Kassam (1979).

\section{RESULTADOS E DISCUSSÃO}

Alguns dos valores médios de $\mathrm{ET}_{\mathrm{c}}$, ao longo do período compreendido entre a poda e a colheita dos frutos, estão apresentados na Tabela 1. Pode-se observar que esses valores variaram de acordo com as condições climáticas predominantes e as fases fenológicas da cultura. A evapotranspiração acumulada ao longo do ciclo produtivo da cultura foi da ordem de 503,0 mm, correspondendo a um valor médio de $4,2 \mathrm{~mm} \mathrm{dia}^{-1}$, enquanto o valor mínimo ocorreu no período entre a poda até 65 dias após, com valor médio de $3,8 \mathrm{~mm} \mathrm{dia}^{-1} ;$ já o valor máximo ocorreu entre 80 e 100 dias após a poda, com um valor médio de $6,5 \mathrm{~mm} \mathrm{dia}^{-1}$; após 100

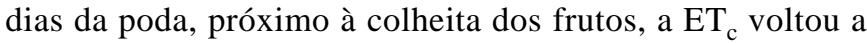
diminuir, atingindo o valor de $4,3 \mathrm{~mm} \mathrm{dia}^{-1}$ e seu valor médio para o ciclo completo mostrou-se dentro dos limites dos valores apresentados por Winkler et al. (1974) e por Dorenbos \& Kassam (1979).

Tabela 1. Variação da evapotranspiração da cultura (ETc) e do coeficiente de cultura $(\mathrm{Kc})$ determinados pelos métodos de Penman-Monteith (ETo(PM)) e do Tanque classe A (CA) ao longo do período entre a poda e a colheita dos frutos na cultura da videira, cv. Itália, em Petrolina, PE, 1994

\begin{tabular}{ccccccc}
\hline DATA & DAP $^{*}$ & ETc & ETo(PM) & ETo(CA) & $\mathrm{kc}(\mathrm{PM})$ & $\mathrm{kc}(\mathrm{CA})$ \\
\hline $03 / 06 / 94$ & 18 & 2,80 & 4,50 & 4,13 & 0,62 & 0,68 \\
$05 / 06 / 94$ & 20 & 4,40 & 5,62 & 6,16 & 0,78 & 0,71 \\
$07 / 06 / 94$ & 22 & 3,60 & 4,76 & 4,41 & 0,76 & 0,82 \\
$09 / 06 / 94$ & 24 & 3,40 & 5,52 & 4,13 & 0,62 & 0,82 \\
$15 / 06 / 94$ & 30 & 3,60 & 5,15 & 4,50 & 0,70 & 0,80 \\
$29 / 06 / 94$ & 44 & 3,90 & 5,49 & 5,46 & 0,71 & 0,71 \\
$30 / 06 / 94$ & 45 & 2,80 & 3,25 & 3,00 & 0,86 & 0,93 \\
$09 / 07 / 94$ & 54 & 3,10 & 5,57 & 5,18 & 0,56 & 0,60 \\
$13 / 07 / 94$ & 58 & 4,10 & 5,31 & 4,90 & 0,77 & 0,84 \\
$14 / 07 / 94$ & 59 & 5,40 & 5,32 & 4,55 & 1,02 & 1,19 \\
$20 / 07 / 94$ & 65 & 4,50 & 5,10 & 4,34 & 0,88 & 1,04 \\
$21 / 07 / 94$ & 66 & 4,10 & 5,61 & 5,25 & 0,73 & 0,78 \\
$11 / 08 / 94$ & 87 & 6,50 & 5,72 & 5,25 & 1,14 & 1,24 \\
$18 / 08 / 94$ & 94 & 7,00 & 6,10 & 6,58 & 1,15 & 1,06 \\
$20 / 08 / 94$ & 96 & 4,50 & 4,78 & 5,04 & 0,94 & 0,89 \\
$21 / 08 / 94$ & 97 & 4,60 & 4,99 & 5,11 & 0,92 & 0,90 \\
$29 / 08 / 94$ & 105 & 5,50 & 6,55 & 6,72 & 0,84 & 0,82 \\
$10 / 09 / 94$ & 116 & 4,30 & 7,19 & 7,42 & 0,60 & 0,58 \\
$11 / 09 / 94$ & 117 & 4,40 & 6,80 & 8,54 & 0,65 & 0,52 \\
\hline * Dias após o plantio & & & & &
\end{tabular}

A Figura 1 apresenta a curva do coeficiente de cultura em relação ao método de Penman-Monteith $(\mathrm{kc}(\mathrm{PM}))$ ao longo dos diferentes estádios fenológicos da videira. $\mathrm{O} \mathrm{kc}$ apresentou valores mínimos $(\mathrm{kc}<0,65)$ no subperíodo de brotação das gemas (até 20 dias após a poda) e máximo $(\mathrm{kc}=1,15)$ nos subperíodos de desenvolvimento e de maturação das bagas ( 80 a 100 dias após a poda). A variabilidade observada entre os valores de $\mathrm{kc}$ obtidos pelos dois métodos propostos, não invalida a aplicação desses coeficientes, desde que se utilize o valor específico para cada método de estimativa de $\mathrm{ET}_{\mathrm{o}}$; assim, a escolha dos valores de $\mathrm{kc}$ obtidos por um ou outro método restringe-se à disponibilidade de parâmetros agrometeorológicos locais. Os valores de kc foram, em geral, superiores aos 
apresentados por Doorenbos \& Kassam (1979) para condições de irrigação complementar, porém são também superiores aos obtidos por Ávila Netto (1997), para a mesma variedade sob irrigação por gotejamento, na região do Submédio São Francisco, que se assemelharam aos resultados obtidos por Doorenbos \& Pruitt (1977) apud Ávila Netto (1997). Deve-se salientar, que os valores de kc mudam também com a variedade, manejo cultural, sistema de irrigação, tipo e cobertura do solo e método de estimativa de $\mathrm{ET}_{\mathrm{o}}$. Oliver \& Sene (1992) obtiveram valores de $\mathrm{kc}$ substancialmente inferiores aos obtidos neste estudo e em Doorenbos \& Kassam (1979). Pode-se justificar tão grande diferença ao se constatar que naquele estudo o parreiral era conduzido no sistema de espaldeira, em que um grande percentual da área fica exposto à radiação solar; por outro lado, o balanço de energia foi realizado de forma a se considerar os componentes sobre plantas e sobre o solo sem vegetação, o que reduziu consideravelmente a energia disponível.

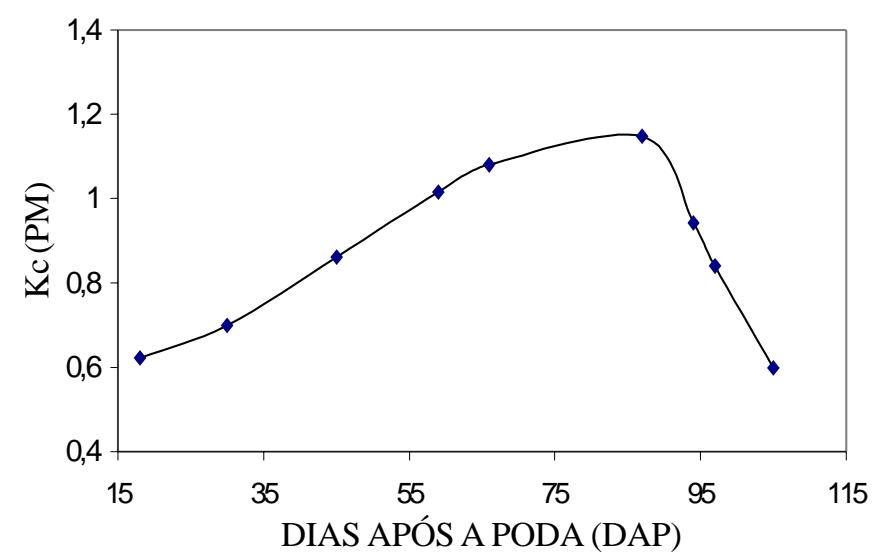

Figura 1. Variação do coeficiente de cultura kc, obtida em relação ao método de Penmam-Monteith, ao longo do período entre a poda e a colheita dos frutos na cultura da videira, cv. Itália, em Petrolina, PE, 1994

\section{CONCLUSÕES}

1. O consumo hídrico da cultura da videira $(503,0 \mathrm{~mm})$ determinado pelo método do balanço de energia, situou-se dentro dos limites estabelecidos em Winkler et al., (1974) e Dorenbos \& Kassam (1979).

2. Os valores dos coeficientes de cultura obtidos experimentalmente, utilizando-se os métodos e condições experimentais descritas são, em geral, superiores aos sugeridos por Dorenbos \& Kassam (1979), e por Ávila Netto (1997).

\section{REFERÊNCIAS BIBLIOGRÁFICAS}

ALLEN, R.G.; SMITH, M.; PERRIER, A.; PERREIRA, L.S. An update for the definition of reference evapotranspiration. ICID Bulletin, New Delhi, v. 43, p.1-34, 1994.

ÁVILA NETTO, J. Necessidades hídricas da videira na região do Submédio São Francisco. Campina Grande: DCA/UFPB. Campina Grande, 1997, 86 p. il. Dissertação Mestrado

BOWEN, I.S. The ratio of heat losses by conduction and by evaporation from any surface. Physical Review, New York, v. 27, p. 779-787, 1926.

DOORENBOS, J.; KASSAM, A.H. Yield response to water. Rome: FAO, 1979, 193p. Irrigation and Drainage Paper 33

HEILMAN, J.L.; McINNES, K.J.; SAVAGE, M.J.; GESH, R.W.; LASCANO, R.J. Soil and canopy energy balances in a west Texas vineyard. Agricultural and Forest Meteorology, Amsterdam, v, 71, p, 99-114, 1994.

MATHIAS, A.D.; COATES, W.E. Wine grape vine radiation balance and temperature modification with fine-mist nozzles. Hort Science, Alexandria-VA, v. 21, p. 1453-1455, 1986.

OLIVER, H.R.; SENE, K.J. Energy and water balances of developing vines. Agricultural and Forest Meteorology, Amsterdam, v. 61, p.167-185, 1992.

SEDIYAMA, G. C. Estimativa da evapotranspiração: Histórico, evolução e análise crítica. Revista Brasileira de Agrometeorologia, Santa Maria, v. 4, n. 1, p. 1-12, 1996.

TANNER, C.B. Energy approach to evapotranspiration from crops. Soil Science of America Proceedings, Madison, v, 24, p, 1-9, 1960.

TEIXEIRA, A.H. de; AZEVEDO, P. V. de. Zoneamento agroclimático para a videira européia no Estado de Pernambuco, Brasil. Revista Brasileira de Agrometeorologia, Santa Maria, v. 4, p.139-145, 1996.

TEIXEIRA, A.H. de; AZEVEDO, P.V. de; SILVA, B.B. da; SOARES, J. M. Balanço de energia na cultura da videira, cv. Itália. Revista Brasileira de Agrometeorologia, Santa Maria, v. 5, p. 137-141, 1997.

WINKLER, A.J.; COOK, J.A.; KLIEWER, W.M.; LIIDER, L.A. General viticulture. 2d. ed. Berkeley: University of California Press., 1974, $710 \mathrm{p}$.

WRIGHT, J. L. New evapotranspiration crop coefficients. Journal of Irrigation and Drainage Division, New York, v. 108, n. IR2, p. 57-74, 1982. 